\title{
Study on Guided Wave Imaging Methods for SOLID Rocket Motor Shell Material
}

\author{
Jian $\mathrm{Li}^{*}$, Xiaofeng Cai, Chunan Ai and Kaixuan Liu \\ Xi'an High-tech Institute, Xi'an Shaanxi, 710025, China \\ ${ }^{*}$ Corresponding author
}

\begin{abstract}
It is easy to produce some defects on the adhesive interface of solid rocket motor, and it is urgent to develop a method on guided wave imaging detection. The propagation characteristics of guided wave dispersion and multi modes are analyzed. According to the propagation path, the guided wave imaging methods can be divided into two types, reflective type and projection type. Three imaging methods which are time reversal focusing, synthetic aperture focusing and transmission tomography are studied, the imaging principle and the research of the domestic and foreign research are analyzed in detail..Different guided wave imaging methods must be selected according to the application requirements.
\end{abstract}

Keywords-SRM; timereversal; synthetic aperture; transmission tomography

\section{INTRODUCTION}

Solid rocket motor (SRM) shell is a kind of bonding structure, which is composed of insulating layer and shell body. The thickness is $3-12 \mathrm{~mm}$. It can be considered as a plate structure, and the bonding interface is the weak link of engine structure. Ultrasonic nondestructive testing technology has comprehensive advantages in the size, location, orientation, depth and property of the internal defects, and the traditional ultrasonic nondestructive testing technology is usually used to enter the test piece, and then analyze the waveform of the interaction between the acoustic wave and the defect in the test piece. But for the plate structure, especially the thickness of the thin plate under the $6 \mathrm{~mm}$ structure, the shear wave or longitudinal wave inspection has become very difficult. When the ultrasonic wave is in the plate structure, the continuous reflection of the two surface boundaries is affected by the structure of the plate. The body wave detection is limited by the coverage of the probe, and it must be carried by point to point, so it takes a long time. Compared to body wave detection, the guide wave detection has obvious advantages, its attenuation is less and the transmission distance is farther. With double probes detection, the received guide wave signal contains the whole information of regions, and the linear region of the plate structure can be detected at each time. In addition, the guided wave has the characteristic of multi mode, and the different modes have different sensitivity to different depth.

Based on the propagation characteristics of guided wave, ultrasonic guided wave imaging detection technology has great development potential in the field of large plate like structure, such as SRM shell, and it is one of the development directions of nondestructive testing technology in the future.

\section{PROPAGATION CHARACTERISTICS}

The propagation characteristics of guided wave are very complex, which mainly manifests in its dispersion characteristic and multi modes phenomenon. As shown in Figure I. The characteristic is that the velocity of the guided wave changes with the frequency. The vibration of the plate wave is constrained by the free boundary of the upper and lower surface, and the resonance wave is formed in the direction of the plate thickness. The plate wave with different vibration modes and vibration frequency is different in the direction. This phenomenon is called frequency dispersion. The forming cause of guided wave is the elastic vibration of the solid slab in the free boundary, and the displacement of the wave propagates along the direction of the wave propagation and the direction of the plate plane.

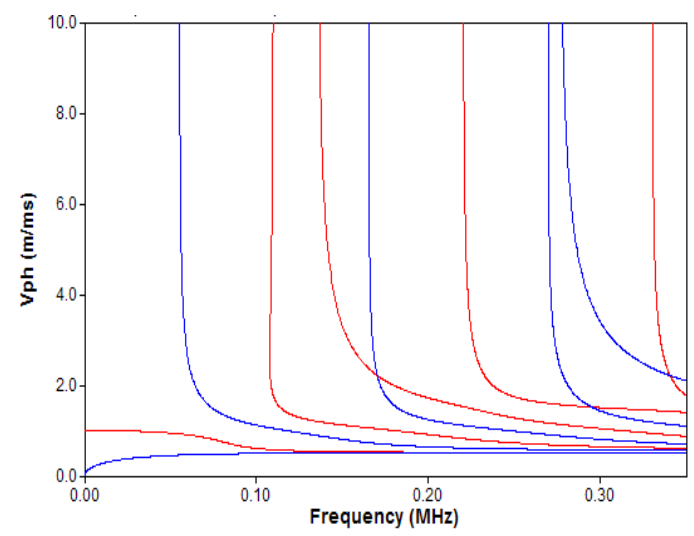

FIGURE I. FREQUENCY DISPERSION CURVE

\section{GUIDED WAVE IMAGING MethodS}

Guided wave imaging according to the characteristics had been made a great progress in guided wave nondestructive testing. It is the developing trend of the non destructive testing to identify the defect location quickly and directly. But it is constrained by the characteristics of multi modes and frequency dispersion, and the application is not very widespread, it is still in the research stage. At present, there are two types of wave imaging detection methods, which are reflection type and transmission type, the reflective type imaging method is realized by the received guided wave information, it mainly include time reversal focusing imaging method and the synthetic aperture focusing imaging method. The transmission type idea originates from the $\mathrm{X}$ ray, such as transmission tomography method. 


\section{A. Time Reversal Focusing Imaging}

Time reversal focusing method based on acoustical reciprocity principle, in which different sensors to receive the sound source emission signal first, then according to the reverse process of the time course emit the signal again, the received signal first to post, and the signal to the first hair, at last the two time wave signal return to source at the same time, and thereby it is possible to reconstruct the sound source signal effectively.

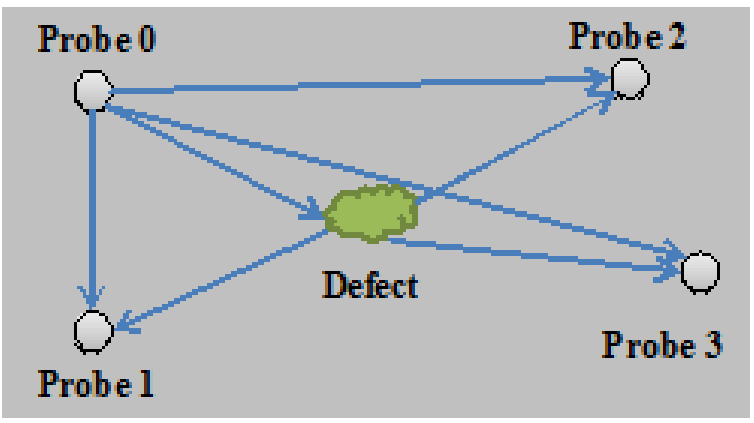

FIGURE II. TIME REVERSAL FOCUSING IMAGING

Time reversal focusing imaging principle is shown in Figure II, where probe 0 is the transmitting probe, and probe $1-3$ is the receiving probe, the intermediate green area is simulated as the defect. In health condition, according to the frequency spectrum of the transfer function between the transmitting probe and the receiving probe, which is determined by the coupling and the piezoelectric effect, the response signal is acquired at each receiving probe, and then made the signal time reversal which performed complex conjugation in the frequency domain and applied to the receiving probe as an excitation signal, at this time, the probe 0 was changed into receiving probe. Through the output response signal we found that transfer function is a real positive and even functions, and it represents an amplification factor which means to focus on the original signal amplification. When there is structural damage, we calculate the response signal similarly. According to the differential signal of the receiving probe between the health state and damage state, the damage signal can be acquired. The defect can be considered as the secondary sources, and the damage scattering signals arrival time of each sensor are different. First made the different time offset delay, and then the signal is up against. After extracting the characteristic values of the focusing signal, and combining the ellipse or other positioning techniques, the distribution of the eigenvalues is associated with the contrast of each pixel in the image. In this way, the defect is visualized.

Professor Victor Giurgiutiu had researched the principle and process of the time reversal focusing[1]. The research result by Professor F. G. Yuan show that this method has good prospects for the health monitoring of complex structures[2]. KULKARNI G and MITRA M had researched the property of time reversal by finite element method[3].

In China, Professor Qu of Wuhan University used guided wave time reversal method to detect defects in aluminum plate. The results show that the two different degree defects can be effectively identify and accurate positioning by guided wave time reversal method. Wang Qiang of Nanjing Aeronautics \& Astronautics University used this method to defect the epoxy glass fiber board. Zhou Jinjie of Beijing Industrial University designed a high voltage pulsed excitation sensor which can transmit time reversal guided wave. Cai Jian researched an virtual time reversal method based on wide band excitation. Qiu Lei researched multi source impact imaging method based on Shannon complex wavelet and time reversal, and the error is not more than $2 \mathrm{~cm}$.

The present research showed that the time reversal focusing method has the spatial and temporal focusing properties, which can make the energy focus on the defects. In the end, the amplitude of the defect echo is greatly increased and the sensitivity of the defect is improved.

\section{B. Synthetic Aperture Focusing Imaging}

Ultrasonic transducers which work in accordance with the scanning- sampling - scanning mode were moved along the horizontal direction, and transmit and receive the echo signal in a constant time interval d, as shown in Figure III. After the scan, the echo signal is processed and the image is constructed.

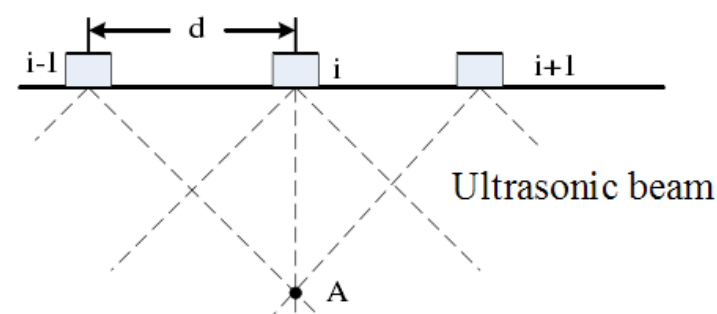

FIGURE III. SYNTHETIC APERTURE FOCUSING IMAGING

The delay of each echo signals are different because the different distances between the reconstruction point and each sampling point, so the phase of each echo signal need to be corrected. In the place where there is a defect, the echo signals are superimposed, and the amplitude increases. In the absence of defects, the echo signal is random, and so weak. Compared with other imaging methods, synthetic aperture imaging method has two notable features:

1) Azimuth resolution is independent of the action distance and the acoustic wavelength.

2) Synthetic aperture imaging method has the near field applicability.

In 1967, John J first proposed the synthetic aperture concept and used it in ultrasonic imaging system[4]. Since then, the application of synthetic aperture in the field of ultrasonic testing has been developed. Edward L.Ginzton Laboratory of Stanford University developed the first digital synthetic aperture imaging system [5]. Levin F. Nock proposed the synthetic receive aperture concept ,then made some simulation and experiment in 1992,It is proved that the resolution ratio is the highest when used the synthetic aperture imaging method[6]. Sverre Holm of the Oslo University in Norway proposed a synthetic transmit aperture imaging methods for improving the system frame in 1997[7]. S.I. Nikolov designed a synthetic aperture focusing beam synthesizer by FPGA, whichs working frequency is up to $167.8 \mathrm{MHz}[8]$. 
In China, the research on the synthetic aperture began comparatively late, and the related research reports were not much. Sun Baoshen and Shen Jianzhong of Chinese Science Academy first introduced the synthetic aperture focusing ultrasonic imaging concept, and analized the basic principle and method, then introduced the relevant receiving technology and the frequency domain processing technology. Liu Dongqing of Scientific acoustics Institute researched the influence of beam directivity by the number of sub aperture, array element spacing and sub aperture. Du Yinghua of Tianjin University simulated the acoustic radiation model and made experiment, the results showed that the synthetic aperture imaging method can improve the imaging system resolution ratio effectively.

\section{Transmission Tomography}

When we take double probes detection, the receiving signal contains the whole information of the region structure between the transmitter and the receiver. A series of guided wave data are obtained by scanning the region along the direction of the detection, and the image of the defect region can be reconstructed by using the projection data of these different directions.

In the fifties of the 20th century, American neurosurgeon Oldendorf published the first article on real significance of CT to overcome ghosting ordinary X-ray imaging of organizational structure, that's called computer tomography. In 1993, Hutchins studied the hole defects in aluminum plate. The results showed that the guided wave signal generated by pulsed laser source and electromagnetic acoustic transducer can be used for structural change tomography of thin plate materials[9]. In 1994, Jansen spread the LWT technology to the field of composite materials nondestructive testing[10]. In 2000, Malyarenko studied the filtered method for fan beam projection and double crosshole geometry[11]. In 2002, Leonard presented a sample of parallel projection and fan beam projection[12].

In China, Ding Xifa designed a kind of ultrasonic tomography detection system based on surrounding array. The ultrasonic probes are placed evenly around the object according to the shap of the specimen, one of which is used to transmit, the rest are used to receive, so the whole specimen can be detected through full range. Song Wen'ai proposed a method to change the ultrasonic array signal into two-dimensional gray level, by this way, the defect image in the material can be reconstructed quickly and accurately. Yang Fengbao proposed a detection method based on micro acoustic excitation and acoustic array, this method not only overcomes the low speed and coupling problem in the detection process, but also greatly reduces the erroneous judgement and misjudge.

High speed, clear and reliable are the development trend of ultrasonic tomography, which means that data acquisition and imaging speed is faster, the reconstructed image has higher spatial resolution and density resolution, and the image is more clear and reliable. To reconstruct image under the condition of data missing is also the problem the CT must solved in the future.

\section{REFERENCE}

[1] Xu B, Giurgiutiu V. Single Mode Tuning Effects on Lamb Wave Time Reversal with Piezoelectric Wafer Active Sensors for Structural Health Monitoring[J]. Journal of Nondestructive Evaluation, 2007, 26: 123-134.

[2] Wang L, Yuan F.G, Damage Identification in a Composite Plate using Prestack Reverse-time Migration Technique[J]. Structural Health Monitoring, 2005, 4 (3): 195-211.

[3] KULKARNI G,MITRA M.Simulation of time reversibility of Lamb wave in symmetric composite laminate[J]. International Journal of Mechanical Sciences,2012,54:277-286.

[4] John J. Flaherty, Elk Grove Village, Kenneth R. Erikson, Niles, Van Metre Lund, Synthetic Aperture Ultrasonic Imaging Systems, 1970, Patent No: 3548642.

[5] G S Kino, D Corl, S Bennett, K Peterson, Real Time Synthetic Aperture Imaging System[J], Ultrasonics Symposium, 1980, 722 731.

[6] Levin F Nock, Gregg E Trahey, Synthetic receive aperture imaging with phase correction for motion and for tissue inhomogeneities-part I : Bacis Principles, IEEE transactions on ultrasonics, 1992, 39(4), 489 495.

[7] Sverre Holm, Hongxia Yao, Improved framrate with synthetic transmit aperture imaging using prefocused subapertures[J], IEEE ultrasonics symposium, 1997, 1535 1538.

[8] Svetoslav Ivanov Nikolov, Jorgen Arendt Jensen, Borislav Gueorguiev Tomov, Fast parametric beamformer for synthetic aperture imaging[J], IEEE transactions on ultrasonics,2008, 55(8), 1755 1767.

[9] Hutchins D A, Jansen D P, Edwards. Lamb wave tomography using non-contact transduction[J]. Ultrasonics, 1993,31(2):97-103.

[10] Jansen D P, Hutchins DA, Mottram JT. Lambwave tomogra-phy of advanced composite laminates containing damage[J].Ultrasonics, 1994,32(2): 83-89.

[11] Malyarenko, Hinders M K. Fan beam and double crosshole lamb wave tomography for mapping flaws aging aircraft struc-tures[J]. J Acoust Soc Am, 2000,108(4): 1631-1639.

[12] Leonard K R, Malyarenko E V, Hinders M K, Ultrasonic Lamb tomography[J]. Inverse Problems, 2002,18: 1795-1808. 